\title{
Systems biology approach to identify alterations in the stem cell reservoir of subcutaneous adipose tissue in a rat model of diabetes: effects on differentiation potential and function
}

\author{
Raquel Ferrer-Lorente • Maria Teresa Bejar • \\ Monica Tous • Gemma Vilahur • Lina Badimon
}

Received: 30 January 2013 / Accepted: 24 September 2013 / Published online: 17 October 2013

(C) Springer-Verlag Berlin Heidelberg 2013

\begin{abstract}
Aims/hypothesis Autologous progenitor cells represent a promising option for regenerative cell-based therapies. Nevertheless, it has been shown that ageing and cardiovascular risk factors such as diabetes affect circulating endothelial and bone marrow-derived progenitor cells, limiting their therapeutic potential. However, their impact on other stem cell populations remains unclear. We therefore investigated the effects of diabetes on adipose-derived stem cells (ASCs) and whether these effects might limit the therapeutic potential of autologous ASCs.

Methods A systems biology approach was used to analyse the expression of genes related to stem cell identification in subcutaneous adipose tissue (SAT), the stromal vascular fraction and isolated ASCs from Zucker diabetic fatty rats and their non-diabetic controls. An additional model of type 2 diabetes without obesity was also investigated. Bioinformatic approaches were used to investigate the biological significance of these changes. In addition, functional studies on cell viability and differentiation potential were performed.

Results Widespread downregulation of mesenchymal stem cell markers was observed in SAT of diabetic rats. Gene expression and in silico analysis revealed a significant effect
\end{abstract}

Electronic supplementary material The online version of this article (doi:10.1007/s00125-013-3081-z) contains peer-reviewed but unedited supplementary material, which is available to authorised users.

R. Ferrer-Lorente $\cdot$ M. T. Bejar $\cdot$ M. Tous $\cdot$ G. Vilahur $\cdot$

L. Badimon $(\bowtie)$

Cardiovascular Research Center, CSIC-ICCC, Hospital de la Santa

Creu i Sant Pau (UAB), C/Sant Antoni Maria Claret 167,

08025 Barcelona, Spain

e-mail: 1badimon@csic-iccc.org

R. Ferrer-Lorente $\cdot$ G. Vilahur $\cdot$ L. Badimon

Centro de Investigación Biomédica en Red de Fisiopatología de la

Obesidad y Nutrición (CIBERObn), Spain

URL: www.ciberobn.com on molecules involved in the maintenance of pluripotency and self-renewal, and on the alteration of main signalling pathways important for stem cell maintenance. The viability and differentiation potential of ASCs from diabetic rats was impaired in in vitro models and in in vivo angiogenesis.

Conclusions/interpretation The impact of type 2 diabetes on ASCs might compromise the efficiency of spontaneous selfrepair and direct autologous stem cell therapy.

Keywords Adipose-derived stem cells · Cell therapy · Differentiation potential - Gene expression and in silico analysis $\cdot$ Pluripotency $\cdot$ Stemness $\cdot$ Subcutaneous adipose tissue $\cdot$ Type 2 diabetes

$\begin{array}{ll}\text { Abbreviations } \\ \text { ASCs } & \text { Adipose-derived stem cells } \\ \text { ASCs }{ }^{\text {ChDMII }} & \text { ASCs from ChDMII rats } \\ \text { ASCs }{ }^{\text {ZDF }} & \text { ASCs from ZDF rats } \\ \text { ASCs }{ }^{\text {ZLC }} & \text { ASCs from ZLC rats } \\ \text { AU } & \text { Arbitrary units } \\ \text { BMPCs } & \text { Bone marrow-derived progenitor cells } \\ \text { ChDMII } & \text { Chemically generated rat model of non-obese } \\ & \text { type 2 diabetes } \\ \text { EC } & \text { Endothelial cell } \\ \text { EPCs } & \text { Endothelial progenitor cells } \\ \text { FGF } & \text { Fibroblast growth factor } \\ \text { IPA } & \text { Ingenuity Pathway Analysis } \\ \text { MSCs } & \text { Mesenchymal stem cells } \\ \text { MTS } & \text { 3-(4,5-Dimethylthiazol-2-yl)-5-(3- } \\ & \text { carboxymethoxyphenyl)-2-(4-sulfophenyl)- } \\ & \text { 2H-tetrazolium, inner salt (assay) } \\ \text { SAT } & \text { Subcutaneous adipose tissue } \\ \text { SVF } & \text { Stromal vascular fraction } \\ \text { ZDF } & \text { Zucker diabetic fatty } \\ \text { ZLC } & \text { Zucker lean normoglycaemic control }\end{array}$




\section{Introduction}

Cell therapy is a promising option for tissue engineering and regenerative medicine. In recent years, numerous animal studies on cell therapy as a potential treatment for ischaemic diseases have been conducted and shown promising results $[1,2]$, in particular studies using bone marrow-derived progenitor cells (BMPCs) [3, 4]. Nevertheless, the translation of such therapies into clinical trials has generated controversy [5, 6]. It has been shown that ageing and cardiovascular risk factors such as diabetes affect endothelial progenitor cells (EPCs) and BMPCs, thereby limiting their therapeutic potential [7-11]. However, the effect of the above risk factors on other stem cell populations remains unclear.

Mesenchymal stem cells (MSCs) were first discovered as clonogenic, adherent colony-forming fibroblastic cells that are present in bone marrow stroma [12]. The key characteristics of MSCs include: adherence to plastic, extensive proliferative capacity, expression of several common cell surface antigens and the ability to differentiate into many lineage-specific cell types $[13,14]$. For many years, BMPCs were considered the major source of stem cells for tissue engineering applications. However, it is now known that MSCs reside in all organs and tissues [15]. Due to their abundance and ease of collection, stem cells isolated from adipose tissue (adipose-derived stem cells [ASCs]) have in recent years generated rapidly growing interest in their developmental plasticity and therapeutic potential [16, 17]. Clinically relevant stem cell numbers can be isolated from adipose tissue, as ASCs have higher proliferation rates than BMPCs [18]. ASCs are an abundant and practical source of donor tissue for autologous cell replacement [19, 20].

Our aim therefore was to investigate the possible effects of diabetes on ASCs and whether these effects might limit the efficiency of autologous ASC transplantation in diabetic patients. Our investigations were performed using two models of diabetes: (1) the Zucker diabetic fatty (ZDF) (Lepr ${ }^{\mathrm{fa} / \mathrm{fa}}$ ) rat, which is a genetic model of type 2 diabetes and obesity [21]; and (2) age-matched Zucker lean normoglycaemic control (ZLC) $\left(\mathrm{Lepr}^{\mathrm{fa} /+} / \mathrm{Lepr}^{+/+}\right)$rats, a second model of biochemically induced diabetes without obesity [22].

\section{Methods}

Animals

All procedures followed in the study fulfilled the criteria of the Guide for the Care and Use of Laboratory Animals published by the US National Institutes of Health (NIH Publication number 85-23, revised 1996) and were approved by the Internal Animal Committee Review Board.
Zucker diabetic fatty rats We purchased 7-week-old male ZDF and ZLC rats from Charles River Laboratories (L'Arbresle Cedex, France). Only ZDF rats had blood glucose levels of $>16.65 \mathrm{mmol} / 1$ from 11 weeks of age (electronic supplementary material [ESM] Fig. 1).

Rat model of non-obese type 2 diabetes We used a chemically generated rat model of non-obese type 2 diabetes (ChDMII) based on ZLC rats, which were made diabetic by a combination of nicotinamide $(230 \mathrm{mg} / \mathrm{kg}$, i.p.) injected $15 \mathrm{~min}$ before streptozotocin (65 mg/kg, i.v.) [22].

Nude Balb/c mice We purchased 8-week-old nude Balb/c mice from Janvier Laboratories (Le Genet-St-Isle, France) and used them for in vivo angiogenesis assays.

A detailed description of the protocols is available in the ESM (Methods, section 1.1).

\section{Serum determinations}

Blood was collected, centrifuged and stored at $-80^{\circ} \mathrm{C}$ for further analysis. Biochemical analyses were determined with an analyser (CLIMA MC-15; RAL, Barcelona, Spain).

\section{Isolation and culture of ASCs}

Subcutaneous adipose tissue (SAT) was excised and processed as detailed in the ESM (Methods, section 1.2) to obtain ASCs.

\section{Flow cytometry}

Freshly collected stromal vascular fraction (SVF) and cultured ASCs were characterised by flow cytometry. A detailed description of the protocol and antibodies used is available in the ESM (Methods, section 1.3).

ASC viability and detection of apoptosis in vitro

Cell viability was evaluated by using the colorimetric 3-(4,5dimethylthiazol-2-yl)-5-(3-carboxymethoxyphenyl)-2-(4sulfophenyl)-2H-tetrazolium, inner salt (MTS) assay (CellTiter $96 \mathrm{AQ}_{\text {ueous }}$ One Solution Cell Proliferation Assay; Promega, Madison, WI, USA). Apoptotic cells were detected using an in situ apoptosis detection kit (ApopTag Red; Chemicon, Temecula, CA, USA) and an annexin V-FITC apoptosis detection kit (BD Biosciences, San Diego, CA, USA). The protocols are described in detail in the ESM (Methods, sections 1.4 and 1.5).

Gene expression and in silico analysis

Total RNA was isolated from SAT and ASCs using kits (SAT: RNeasy lipid tissue kit, ASCs: RNeasy mini isolation kit, both 
from Qiagen, Valencia, CA, USA) according to the manufacturer's instructions. cDNA was synthesised from $1 \mu \mathrm{g}$ total RNA using a high-capacity cDNA archive kit (Applied Biosystems, Foster City, CA, USA) and analysed by real-time PCR using the Prism 7900HT Sequence Detection System (Applied Biosystems) according to the manufacturer's instructions. mRNA expression in ASCs and SAT was normalised against the average expression of two reference genes, Rplp0 and $R p l 13 a$, the stability of whose expression was validated using geNorm version 3.5 software (http://medgen.ugent.be/ genorm). The respective cycle threshold $\left(\mathrm{C}_{t}\right)$ values in ZLC were 20.12 and 24.5 for SAT, and 17.59 and 18.4 for ASCs. The mean expression of reference genes differed between ZLC and ZDF rats by factors of 1.04 and 1.26 in ASCs and SAT, respectively. mRNA expression in ASCs after adipogenic, osteogenic and endothelial differentiation was normalised against the expression of the reference gene $R p l p 0$, which differed in cells of ZLC and ZDF rats by factors of $0.65,0.72$ and 1.0, respectively. TaqMan gene expression assays and sequences of the primers used are provided in ESM Table 1.

Gene expression in the SVF was analysed by a specific array (Rat Stem Cell PCR; SA Biosciences, Frederick, MD, USA), using pooled material due to the limited number of samples. The contribution of each sample was equal. A detailed description of the protocols is available in the ESM (Methods, section 1.6).

The Ingenuity Pathway Analysis (IPA) software package (Ingenuity Systems, www.ingenuity.com) was used to gain insight into biological functions and pathways represented by the differentially expressed transcripts in each group.

\section{In vitro ASC differentiation}

Adipogenic, osteogenic and endothelial cell (EC) differentiation assays were performed using specific differentiation media. Detailed protocols can be found in the ESM (Methods, section 1.7).

Angiogenesis assay in vivo

Nude mice ( 8 weeks old) were injected subcutaneously with $4 \times 10^{6}$ undifferentiated ASCs diluted in $400 \mu \mathrm{l}$ cold, growth factor-reduced Matrigel (BD Biosciences). As a control, Matrigel without cells was also injected. After 7 days, mice were killed and Matrigel plugs were removed, photographed and processed for histological analysis or haemoglobin assessment. For detailed methods, see ESM (Methods sections 1.8 and 1.9).

Statistical analyses

Results are expressed as mean \pm SEM. Student's $t$ test or one-way ANOVA followed by Bonferroni's post hoc test were used for statistical analysis using a software package (GraphPad, La Jolla, CA, USA). A value of $p<0.05$ was considered significant.

IPA software uses right-tailed Fisher's exact test to calculate the $p$ value, thus determining the probability that biological functions are affected. The regulation $z$ score algorithm is used to make predictions on whether functions should increase or decrease given the observed gene expression changes. The $z$ score algorithm is designed to reduce the chance that random data will generate significant predictions. An absolute $z$ score of $\geq 2$ is considered significant.

\section{Results}

Metabolic profile

Metabolic profiles of ZDF and ZLC rats are detailed in ESM Table 2. ZLC rats had normal blood glucose levels (5.13 \pm $0.11 \mathrm{mmol} / \mathrm{l}$ ), whereas those of ZDF rats were significantly higher $(21.80 \pm 0.64 \mathrm{mmol} / \mathrm{l}, p<0.0001 \mathrm{vs}$ controls), clearly reflecting their diabetic status (ESM Fig. 1). Significantly increased levels of total cholesterol, triacylglycerol, urea and glutamic pyruvic transaminase were also observed in ZDF compared with ZLC rats.

Primary culture of ASCs increases specific stem cell markers

Results revealed the presence of low numbers of stem cells in the SVF. After in vitro culture (passage 3), most adherent cells expressed CD29 (over 95\%), CD90 (over 94\%) and CD44 (44-60\%), and were negative for the haematopoietic marker CD45 (0.2\%). No differences between groups were observed (ESM Table 3).

ASCs from ZDF rats exhibit loss of viability

Loss of viability was observed during in vitro culture of ASCs from $\mathrm{ZDF}$ rats $\left(\mathrm{ASCs}{ }^{\mathrm{ZDF}}\right)$. Flow cytometric analysis revealed a significant decrease in the percentage of live $\mathrm{ASCs}{ }^{\mathrm{ZDF}}$ cells compared with ASCs from ZLC rats $\left(\mathrm{ASCs}^{\mathrm{ZLC}}\right.$ ) (ESM Fig. 2a). To confirm our observations, the viability of ASCs was measured. The MTS assay showed that the number of viable cells in $\mathrm{ASCs}^{\mathrm{ZDF}}$ cultures was significantly lower than in $\mathrm{ASCs}^{\mathrm{ZLC}}$ cultures (ESM Fig. 2b). To determine whether this difference was due to an increased incidence of cell death, apoptosis assays were performed. A significantly increased percentage of apoptotic cells was detected in $\mathrm{ASCs}^{\mathrm{ZDF}}$ cultures compared with ASCs ${ }^{\mathrm{ZLC}}$ (ESM Fig. 2c). The Annexin V-FITC apoptosis detection kit also showed an increase in apoptotic cells (ESM Fig. 2d). 
The expression of genes that define stemness is significantly decreased in ASCs ${ }^{\mathrm{ZDF}}$

Considering the possible effects of diabetes on ASC characteristics, we investigated the expression of genes related to stem cell identification, maintenance and growth. To confirm the effects on ASC content and that the differences observed between ASCs were due to the effects of diabetes and not induced by culture conditions, we analysed the gene expression profiles of SAT and the SVF. Most of the genes analysed were downregulated in diabetic animals.

Gene expression analysis of ASCs for MSC markers (Cd29 [also known as Itgb1], Cd44, Cd73 [also known as Nt5e], Cd90 [also known as Thy1], Cd105 [also known as Eng], Cd166 [also known as Alcam] and collagen type $\alpha 1$ [Colla1]) revealed no significant differences between both groups, although, interestingly, ASCs ${ }^{\text {ZDF }}$ had widespread decreased expression of these markers (Fig. 1). The analysis performed on tissue revealed that diabetes was associated with an overall downregulation of MSC-specific markers. Statistically significant reductions were found for $\mathrm{Cd} 44, \mathrm{Cd} 90, \mathrm{Cd} 105$, Cd166 and Collal (Fig. 2).

The expression of genes that define stemness markers was severely perturbed in diabetic SAT, just as in the derived ASCs, highlighting the decreased expression of Pou5f1. Other genes involved in maintaining pluripotency and selfrenewal showed significantly reduced expression in diabetic samples, e.g. Fgf4 and Podxl in ASCs (Fig. 1), and Dnmt3b, Fgf2, Sox2, Tert and Podxl in SAT (Fig. 2). Moreover, changes in genes encoding molecules related to signalling pathways important for stem cell maintenance (Notch, Wnt and Hedgehog pathways) and linked to cellular movement (Cxcl12 and Cxcr4) were observed. These changes reached significance for Dll1 and Notch2 in ASCs (Fig. 1), and for Notch1, Notch2 and Cxcl12 in tissue (Fig. 2).

SVF gene expression analysis showed the same pattern. We observed a widespread downregulation of stem cell markers in this compartment (ESM Fig. 3), again suggesting that the pluripotent capacity was attenuated.

In silico analysis shows the impact of diabetes on pluripotency, self-renewal and the differentiation potential of ASCs

To identify molecular pathways and functional assignments that differ between the two groups, thus allowing a biological interpretation of these differences, the lists of genes were uploaded into the IPA software. IPA highlighted the implication of downregulation of these genes in cell cycle progression, cellular growth and proliferation, cellular movement, and cardiovascular system development and function. The analysis made it possible to detect related genes, and subsequently analysed and confirmed that genes involved in the maintenance of pluripotency and self-renewal pathways were

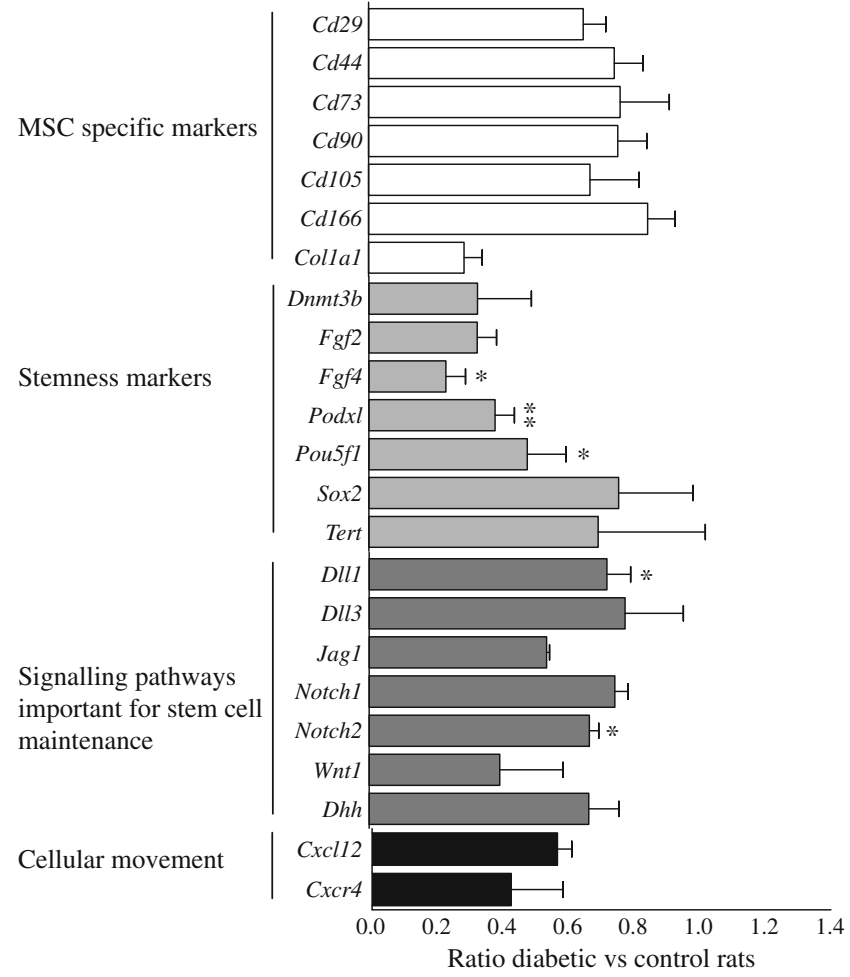

Fig. 1 Gene expression profile of cultured ASCs after three passages. The presence of stem cell-specific markers in subcutaneous ASCs was analysed by real-time PCR. Each data point is presented as the ratio of $\mathrm{ASCs}^{\mathrm{ZDF}}: \mathrm{ASCs}^{\mathrm{ZLC}} ; n=5-6$ per group, in technical duplicates; ${ }^{*} p<0.05$ and $* * p<0.01$ for $\mathrm{ASCs}^{\mathrm{ZDF}}$ vs ASCs ${ }^{\mathrm{ZLC}}$

downregulated in diabetic samples (Tables 1 and 2, ESM Fig. 4).

In agreement with our functional observations in cell viability assays, IPA predicted a decrease of proliferation-related $\left(p=2.22 \times 10^{-11}, z\right.$ score -2.232$)$ and an increase of apoptosisrelated $\left(p=9.56 \times 10^{-9}, z\right.$ score 2.718$)$ gene expression in $\mathrm{ASCs}^{\mathrm{ZDF}}$ compared with ASCs ${ }^{\mathrm{ZLC}}$.

Changes to the in vitro differentiation potential of ASCs ${ }^{\text {ZDF }}$

Adipogenic differentiation Morphological changes associated with adipogenesis were faster and higher in ASCs ${ }^{\mathrm{ZDF}}$, as also evidenced by a marked increase in Herxheimer staining (Fig. 3a). The microscopic observations were confirmed by expression levels of adipogenic markers (Fig. 3b). Both cell types were able to differentiate into adipocytes, showing significant increases in expression of the adipocyte markers Lpl, Fabp4, Cd36 and Pparg. However, differentiated $\mathrm{ASCs}^{\mathrm{ZDF}}$ had higher expression of $\mathrm{Lpl}(p=0.0012), \mathrm{Fabp} 4$ $(p=0.0405), C d 36(p=0.0201)$ and Pparg $(p=$ NS) than differentiated ASCs ${ }^{\text {ZLC }}$. This pattern of expression showed that the adipogenic differentiation potential of $\mathrm{ASCs}^{\mathrm{ZDF}}$ was higher than that of $\mathrm{ASCs}^{\mathrm{ZLC}}$. 


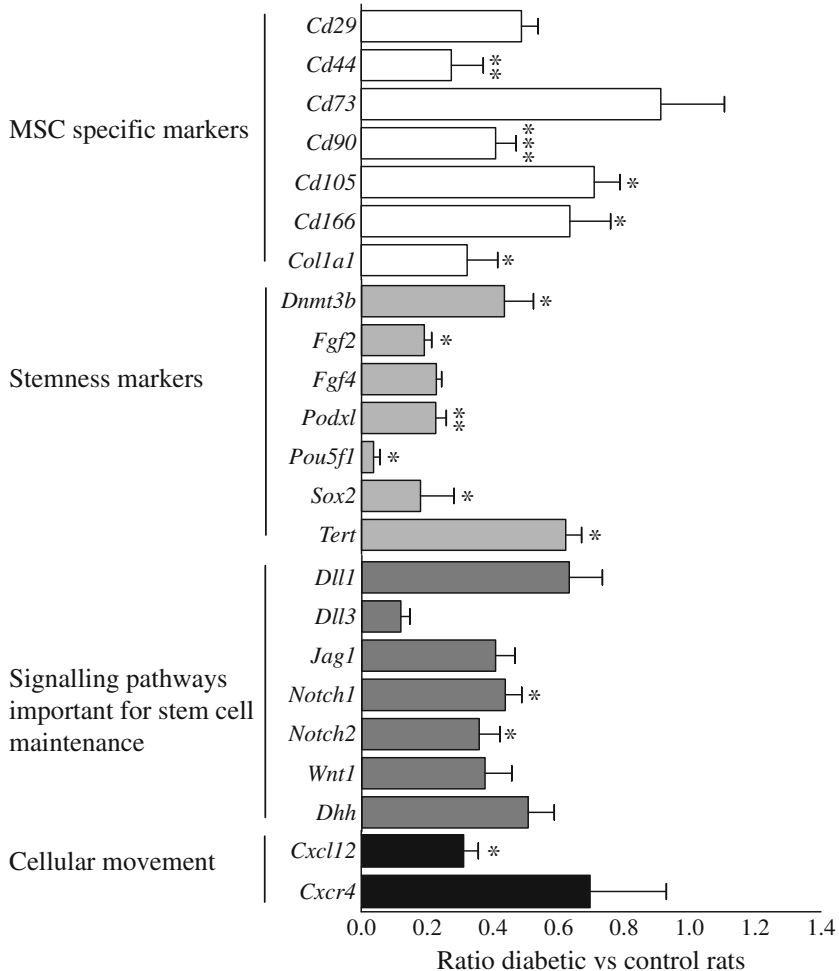

Fig. 2 Gene expression analysis of SAT. The presence of stem cellspecific markers in SAT was analysed by real-time PCR. Each data point is presented as the ratio of ZDF:ZLC; $n=5-6$ per group, in technical duplicates; ${ }^{*} p<0.05, * * p<0.01$ and $* * * p<0.001$ for ZDF vs ZLC

Osteogenic differentiation Real-time PCR analysis of lineagespecific genes indicated differences in osteogenic differentiation between $\mathrm{ASCs}^{\mathrm{ZDF}}$ and $\mathrm{ASCs}{ }^{\mathrm{ZDF}}$. Both cell types were able to differentiate (Fig. 4a, b), showing significantly increased expression of genes encoding the osteogenic markers osteopontin (Spp1), alkaline phosphatase 1 (Alpl) and osteocalcin (Bglap) (Fig. 4b). Thus, in contrast to adipogenic differentiation, $\mathrm{ASCs}^{\mathrm{ZDF}}$ had reduced osteogenic potential.

Endothelial cell differentiation To investigate whether diabetes affects EC differentiation potential, we measured the expression of specific markers in ASCs and capillary-like tube formation in Matrigel. ASCs ${ }^{\mathrm{ZLC}}$ and $\mathrm{ASCs}^{\mathrm{ZDF}}$ showed increased expression of Pecam1 after differentiation (ASCs ${ }^{\mathrm{ZLC}}$ $\left.p<0.0001, \mathrm{ASCs}^{\mathrm{ZDF}} p=0.0109\right)$, whereas the expression of Cd105 only increased significantly in ASCs ${ }^{\text {ZLC }}$ (Fig. 5a). Although both differentiated ASC groups were able to develop capillary-like networks (Fig. 5b), the ability of ASCs ${ }^{\text {ZDF }}$ was significantly impaired compared with that of ASCs ${ }^{\mathrm{ZLC}}$ (Fig. 5c). To study in-depth the angiogenic potential of ASCs, we measured the expression of genes encoding the antiangiogenic factors endostatin (Coll 8 a1), thrombospondin-1 (Thbs1) and serpin peptidase inhibitor, clade E (Serpine1) (ESM Fig. 5a). Interestingly, ASCs ${ }^{\mathrm{ZDF}}$ had significantly higher expression levels of endostatin $(p=0.0311)$ and a non-significant increase in expression of Thbs 1 and Serpine1.
On the other hand, when ASCs were differentiated the expression of anti-angiogenic factors tended to decrease, reaching significance for Serpine1 in both types of cells (ESM Fig. 5b).

Angiogenesis in vivo

Macroscopic observations of the retrieved plugs at day 7 postimplant showed angiogenesis in both types of ASC (Fig. 6a). However, haemoglobin quantification (Fig. 6b) and histological examination (Fig. 6c) revealed that the ability to stimulate microvessel growth was impaired in $\mathrm{ASC}^{\mathrm{ZDF}}$. The haemoglobin content was only significantly increased in the $\mathrm{ASCs}^{\mathrm{ZLC}}$ plugs with respect to control plugs (without ASCs) $(p=0.0239)$. A higher level of von Willebrand factor was also evident in the plugs containing $\mathrm{ASCs}^{\mathrm{ZLC}}$.

Impaired function in $\mathrm{ASCs}^{\mathrm{ZDF}}$ is not due to leptin receptor deficiency

ASCs from ChDMII rats (ASCs ${ }^{\text {ChDMII }}$ ) were also investigated to distinguish between the impact of diabetes and that of leptin receptor deficiency on ASCs. Gene expression profiles for $\mathrm{ASCs}^{\mathrm{ChDMII}}$ and $\mathrm{ASCs}{ }^{\mathrm{ZDF}}$ were similar. Only the expression of Cd166 differed significantly between ASCs ${ }^{\mathrm{ChDMII}}$ and $\mathrm{ASCs}^{\mathrm{ZDF}}$. The expression of Fgf2 and Pou $5 f 1$ was more significantly affected in ASCs ${ }^{\text {ChDMII }}$ than in ASCs ${ }^{\text {ZDF }}$ compared with controls (ESM Fig. 6).

In vitro differentiation assays confirmed that the differentiation potential in ASCs ${ }^{\mathrm{ChDMII}}$ and $\mathrm{ASCs}{ }^{\mathrm{ZDF}}$ was equal. No significant differences in differentiation markers were observed between ASCs ${ }^{\mathrm{ChDMII}}$ and ASCs ${ }^{\mathrm{ZDF}}$ (Fig. 7). As with $\mathrm{ASCs}^{\mathrm{ZDF}}$, the adipogenic potential of ASCs ${ }^{\mathrm{ChDMII}}$ was higher than that of ASCs ${ }^{\mathrm{ZLC}}$, showing significantly higher expression of the adipocyte markers $\mathrm{Lpl}, \mathrm{Fabp} 4$ and $\mathrm{Cd} 36$; in contrast, osteogenic and EC differentiation capacity was reduced. Differentiated ASCs ${ }^{\text {ChDMII }}$ only showed a significant increase in expression of the osteogenic marker Spp1, but this increase was significantly lower than in differentiated $\mathrm{ASCs}^{\mathrm{ZLC}}$. No significant increase of EC markers was observed in differentiated $\mathrm{ASCs}^{\mathrm{ChDMII}}$. Differentiated ASCs ${ }^{\text {ChDMII }}$ were able to develop capillary-like networks of similar length to ASCs ${ }^{\text {ZDF }}$ $\left(\mathrm{ASCs}^{\mathrm{ChDMII}} 2802 \pm 636\right.$ arbitrary units [AU]; $\mathrm{ASCs}^{\mathrm{ZDF}}$ $3104 \pm 580 \mathrm{AU})$, but these were significantly smaller than in $\mathrm{ASCs}^{\mathrm{ZLC}}(5507 \pm 637 \mathrm{AU}, p=0.0310)$.

\section{Discussion}

Autologous progenitor cells represent a novel treatment option for ischaemic complications requiring therapeutic revascularisation and vascular repair. One of the main groups of patients who could benefit from cellular therapy is patients 
Table 1 Principal functions affected by diabetes

\begin{tabular}{|c|c|c|c|}
\hline Category & Function & $p$ value & Top molecules associated \\
\hline Cellular growth and proliferation & Proliferation of cells & $2.22 \times 10^{-11}$ & $\begin{array}{l}\text { CD44, COL1A1, CXCL12, DLL1, DNMT3B, CD105, } \\
\text { FGF2, FGF4, NOTCH1, NOTCH2, POU5F1, SOX2, } \\
\text { TERT }\end{array}$ \\
\hline Cellular growth and proliferation & Growth of cells & $1.45 \times 10^{-9}$ & $\begin{array}{l}\text { CD44, CXCL12, DLL1, DNMT3B, CD105, FGF2, } \\
\text { FGF4, NOTCH1, NOTCH2, POU5F1, SOX2, TERT }\end{array}$ \\
\hline Cell cycle & Cell cycle progression & $1.12 \times 10^{-8}$ & $\begin{array}{l}\text { CD44, COL1A1, CXCL12, FGF2, NOTCH1, } \\
\text { NOTCH2, POU5F1, SOX2, TERT }\end{array}$ \\
\hline Cell cycle & Arrest in cell cycle progression & $4.13 \times 10^{-7}$ & COL1A1, NOTCH1, NOTCH2, POU5F1, SOX2 \\
\hline Cell death & Apoptosis & $9.56 \times 10^{-9}$ & $\begin{array}{l}\text { CD44, CXCL12, DLL1, DNMT3B, FGF2, NOTCH1, } \\
\text { NOTCH2, POU5F1, TERT }\end{array}$ \\
\hline Cell morphology & Tubulation of cells & $1.52 \times 10^{-9}$ & CD166, CXCL12, FGF2 \\
\hline Cellular movement & Transmigration of cells & $4.09 \times 10^{-8}$ & CD44, CXCL12, CD90 \\
\hline Cellular movement & Migration of cells & $2.33 \times 10^{-7}$ & $\begin{array}{l}\text { CD166, CD44, COL1A1, CXCL12, DNMT3B, CD105, } \\
\text { FGF2, PODXL, CD90 }\end{array}$ \\
\hline Cellular movement & Chemotaxis of endothelial cells & $1.82 \times 10^{-6}$ & CXCL12, FGF2, \\
\hline Cellular movement & Mobilisation of stem cells & $6.11 \times 10^{-6}$ & CXCL12 \\
\hline Cell-to-cell signalling and interaction & Binding of cells & $1.87 \times 10^{-7}$ & CD44, CD166, CXCL12, FGF2, NOTCH1 \\
\hline Cellular development & Differentiation of cells & $3.59 \times 10^{-9}$ & $\begin{array}{l}\text { CXCL12, DLL1, CD105, FGF2, FGF4, NOTCH1, } \\
\text { POU5F1, SOX2, TERT }\end{array}$ \\
\hline Cellular development & Differentiation of endothelial cells & $1.33 \times 10^{-4}$ & FGF2, NOTCH1 \\
\hline Cellular development & Endothelial cell development & $6.82 \times 10^{-7}$ & CD44, CD105, FGF2, NOTCH1 \\
\hline Tissue development & Tissue development & $5.06 \times 10^{-8}$ & $\begin{array}{l}\text { CD44, COL1A1, CXCL12, DLL1, CD105, FGF2, } \\
\text { NOTCH1 }\end{array}$ \\
\hline Tissue development & Osteogenesis & $2.92 \times 10^{-3}$ & $\begin{array}{l}\text { CD44, COL1A1, CXCL12, DLL1, CD105, FGF2, } \\
\text { NOTCH1 }\end{array}$ \\
\hline $\begin{array}{l}\text { Cardiovascular system development } \\
\text { and function }\end{array}$ & Development of blood vessel & $5.37 \times 10^{-13}$ & $\begin{array}{l}\text { CD166, CD44, COL1A1, CXCL12, CD105, FGF2, } \\
\text { NOTCH1, TERT }\end{array}$ \\
\hline $\begin{array}{l}\text { Cardiovascular system development } \\
\text { and function }\end{array}$ & Vasculogenesis & $4.58 \times 10^{-12}$ & $\begin{array}{l}\text { CD166, CD44, CXCL12, CD105, FGF2, NOTCH1, } \\
\text { TERT }\end{array}$ \\
\hline $\begin{array}{l}\text { Cardiovascular system development } \\
\text { and function }\end{array}$ & Angiogenesis & $2.14 \times 10^{-7}$ & CD166, CXCL12, CD105, FGF2, TERT \\
\hline
\end{tabular}

Gene expression data were visualised in the context of biological function using IPA software (Ingenuity System, www.ingenuity.com). The table shows the principal functions of the 70 biological functions significantly associated with the molecules analysed. The function analysis of IPA was generated on the basis of all currently available published data

$p$ values are from Fisher's exact test right-tailed

Protein abbreviations: COL1A1, collagen type $\alpha 1$; CXCL12, chemokine (C-X-C motif) ligand 12; DLL1, delta-like 1; DNMT3B, DNA (cytosine-5-)methyltransferase $3 \beta$; PODXL, podocalyxin-like protein 1; POU5F1, POU domain class 5 transcription factor 1; SOX2, SRY (sex-determining region Y) box 2; TERT, telomerase reverse transcriptase

with diabetic complications, yet broadly dysfunctional cells may limit the feasibility of this approach. As many of these cells are being considered for clinical therapies, it is essential to validate the identity and potency of these cells.

The link between diabetes and impaired properties of EPCs and BMPCs is well established [7-10]. However, the impact of diabetes on other types of stem cell remains unclear. In this study, we analysed the effect of the presence of diabetes on ASCs. We compared ASCs from a rat model of diabetes and from age-matched non-diabetic rats, analysing their gene expression profiles, in vitro viability and differentiation potential. To confirm that the differences observed between both groups of ASCs were due to the effects of diabetes and rule out the possibility that changes were induced by in vitro culture and clonal expansion, we analysed the differences between animals in gene expression profiles of SAT and the SVF. As in any gene expression study, the selection of a valid endogenous control is critical to avoid misinterpretation of results. Thus, the suitability of endogenous control genes was evaluated in all experimental groups under comparison, 
Table 2 Top modified canonical pathways in ZDF compared with ZLC rats

Top canonical pathways

\begin{tabular}{ll}
\hline Pathway & $p$ value \\
\hline Notch signalling & $1.76 \times 10^{-9}$ \\
Human embryonic stem cell pluripotency & $1.06 \times 10^{-6}$ \\
Leucocyte extravasation signalling & $6.16 \times 10^{-6}$ \\
Role of Oct4 in mammalian embryonic & $3.71 \times 10^{-5}$ \\
$\quad$ stem cell pluripotency & $9.22 \times 10^{-5}$ \\
Wnt- $\beta$-catenin signalling & $6.61 \times 10^{-3}$ \\
FGF signalling & \\
\hline
\end{tabular}

Gene expression data were visualised in the context of biological function using IPA software (Ingenuity System, www.ingenuity.com). The table shows the top canonical pathways affected by diabetes; 26 pathways were significantly mapped. The pathway analysis of IPA was generated on the basis of all currently available published data

$p$ values from Fisher's exact test right-tailed

confirming that the reference genes did not show a systematic bias that was causally related to the inherent diabetic phenotype. The $C_{t}$ of endogenous genes showed no systematic variation in the ZDF compared with the ZLC group; in all a
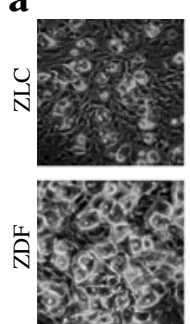

1 week
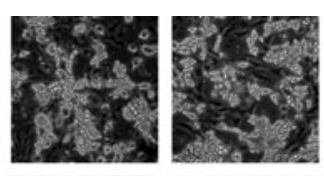

Differentiated Undifferentiated
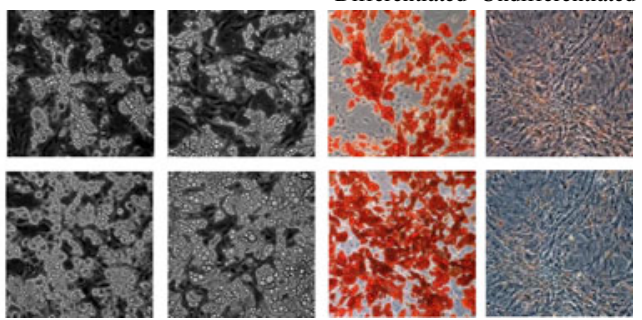

2 weeks

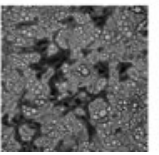

3 weeks
Weeks in culture

b

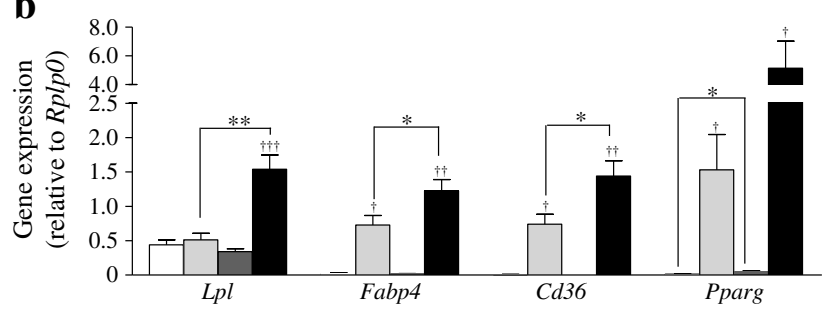

Fig. 3 Adipogenic differentiation of $\mathrm{ASCs}^{\mathrm{ZDF}}$ and $\mathrm{ASCs}^{\mathrm{ZLC}}$. ASCs isolated from $Z D F$ rats showed greater adipogenesis than those from ZLC rats. (a) The evolution of morphological changes using a brightfield microscope (magnification $\times 20$ ). Differentiation potency was evaluated by Herxheimer staining and (b) by lineage-specific gene expression patterns determined by real-time PCR using specific markers $(\mathrm{Lpl}$, Fabp4, Cd36 and Pparg). White bars, undifferentiated ASCs ${ }^{\text {ZLC }}$; dark grey bars, undifferentiated $\mathrm{ASCs}{ }^{\mathrm{ZDF}}$; light grey bars, differentiated $\mathrm{ASCs}^{\mathrm{ZLC}}$; black bars, differentiated ASCs ${ }^{\mathrm{ZDF}}$. Each data point (b) is presented as gene expression relative to the reference gene. The results are means \pm SEM; $n=5-6$ per group, in technical duplicates; ${ }^{*} p<0.05$ and $* * p<0.01$ for ASCs ${ }^{\mathrm{ZDF}}$ vs ASCs ${ }^{\mathrm{ZLC}} ;{ }^{\dagger} p<0.05,{ }^{\dagger \dagger} p<0.01$ and ${ }^{\dagger \dagger} p<0.001$ for differentiated vs undifferentiated cells $\mathbf{a}$
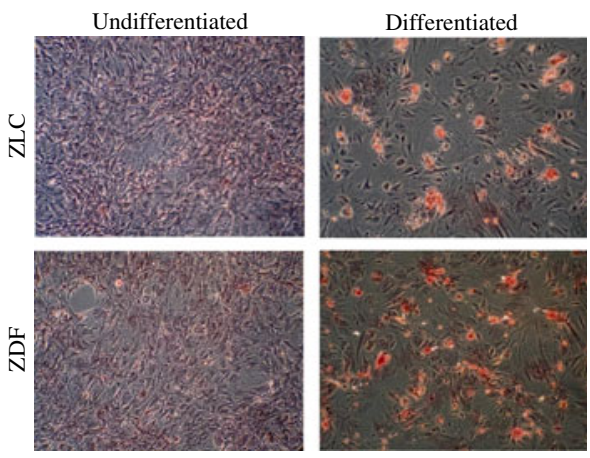

b

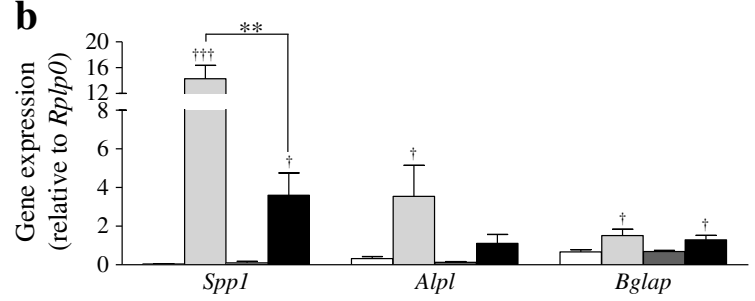

Fig. 4 Osteogenic differentiation of ASCs ${ }^{\mathrm{ZDF}}$ and $\mathrm{ASCs}^{\mathrm{ZLC}}$. (a) Differentiation potency was evaluated by Alizarin red staining and (b) by lineage-specific gene expression patterns determined by real-time PCR using specific markers (Spp1, Alpl and Bglap). White bars, undifferentiated $\mathrm{ASCs}^{\mathrm{ZLC}}$; dark grey bars, undifferentiated $\mathrm{ASCs}^{\mathrm{ZDF}}$; light grey bars, differentiated ASCs ${ }^{\text {ZLC }}$; black bars, differentiated ASCs ${ }^{\mathrm{ZDF}}$. Each data point (b) is presented as gene expression relative to the reference gene. The results are means $\pm \mathrm{SEM} ; n=5-6$ per group, in technical duplicates; $* * p<0.01$ for $\mathrm{ASCs}^{\mathrm{ZDF}}$ vs $\mathrm{ASCs}^{\mathrm{ZLC}}{ }^{*} p<0.05$ and ${ }^{\dagger \dagger} p<0.001$ for differentiated vs undifferentiated cells

cases, moreover, the small variability that we did observe $\left(\Delta \mathrm{C}_{\mathrm{t}}<0.5\right)$ was the common variability associated with the procedure [23].

$\mathrm{ASCs}^{\mathrm{ZDF}}$ cultures showed a significant worsening in the course of time. To confirm our observations, viability and apoptosis assays were performed, showing impaired viability in $\mathrm{ASCs}^{\mathrm{ZDF}}$ compared with $\mathrm{ASC}^{\mathrm{ZLC}}$. Previous studies had shown increased apoptotic activity of MSCs after exposure in vitro to added high glucose concentrations [24].

In agreement with several studies [15, 18, 19], ASCs were positive for the MSC marker genes $C d 29, C d 44, C d 73$, Cd90, Cd105, Cd166 and Colla1. Interestingly, although gene expression analysis failed to reveal significant differences between the two groups, ASCs ${ }^{\text {ZDF }}$ had decreased expression for these surface markers. The analysis performed for genes related to stem cell identification in SAT and the SVF revealed overall downregulation, possibly indicating that diabetes reduces the stem cell reservoir in SAT.

IPA enables gene expression data to be displayed in a systems biology context, showing a significant effect on signalling pathways important for stem cell maintenance. Results revealed a clear effect of diabetes on molecules involved in maintenance of pluripotency and self-renewal, highlighting the significant decreased expression of Pou5fl, which encodes an essential factor for maintenance of the pluripotent 
a

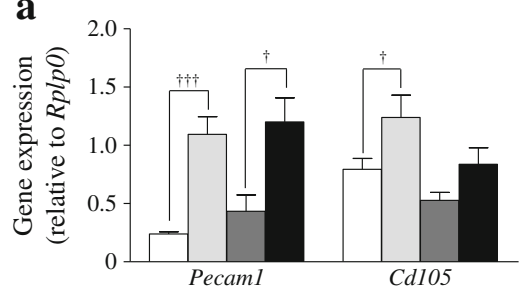

$\mathbf{b}$

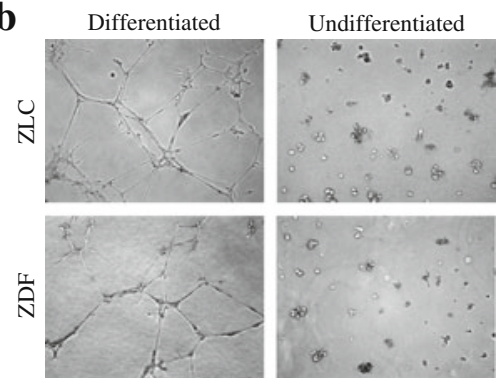

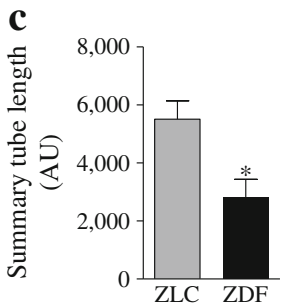

Fig. 5 Differentiation of $\mathrm{ASCs}^{\mathrm{ZDF}}$ and $\mathrm{ASCs}^{\mathrm{ZLC}}$ into ECs. (a) The acquisition of an endothelial phenotype was evaluated by specific endothelial marker genes (Pecam1 and Cd105) using real-time PCR and (b) by a Matrigel in vitro tube formation assay. (a) Each data point is presented as gene expression levels relative to the reference gene. White bars, undifferentiated $\mathrm{ASCs}^{\mathrm{ZLC}}$; dark grey bars, undifferentiated $\mathrm{ASCs}^{\mathrm{ZDF}}$; light grey bars, differentiated ASCs ${ }^{\mathrm{ZLC}}$; black bars, differentiated $\mathrm{ASCs}^{\mathrm{ZDF}}$. (c) Quantification of total length of the tubes formed by differentiated ASCs. (a, c) The data are means \pm SEM; $n=5-6$ per group, (a) in technical duplicates; $* p<0.05$ for $\mathrm{ASCs}^{\mathrm{ZDF}}$ vs ASCs ${ }^{\mathrm{ZLC}} ;{ }^{\dagger} p<0.05$ and ${ }^{\dagger \dagger} p<0.001$ for differentiated vs undifferentiated cells

state and for self-renewal in stem cells [25]. This transcription factor is not only required to maintain pluripotency in the developing embryo [26], but is also, as shown by several studies, associated with the undifferentiated pluripotent state of stem cell populations derived from adult tissues, as well as playing a role as reprogramming factor to induce pluripotent stem cells $[27,28]$.

Of the 26 pathways significantly mapped, Notch signalling was the top canonical pathway affected by diabetes. Notch signalling is implicated in the maintenance of self-renewal potential in stem cells [29]; its activation in stem cells leads to the maintenance of self-renewal potential through downregulation of tissue-specific transcription factors, while its inhibition induced terminal differentiation [30]. Notch was shown to play a role in adipocyte differentiation from MSCs [30-33]. However, conflicting findings have been reported concerning its role. It has been argued that Notch is dispensable in adipocyte differentiation [30]; but other evidence shows that inhibition of Notch signalling or its target genes can inhibit adipocyte differentiation [31] or, in contrast, that Notch inhibition enhances adipogenesis $[32,33]$. Here, we show an obvious inhibition of Notch signalling in ASCs ${ }^{\text {ZDF }}$, which in agreement with the results of Huang et al [33] and Ugarte et al [32], would explain the overexpression of Pparg in undifferentiated $\mathrm{ASCs}{ }^{\mathrm{ZDF}}$ and their higher adipogenic differentiation potential.
Our analysis also disclosed an effect on the Wnt- $\beta$-catenin signalling pathway. Wnt signalling maintains pre-adipocytes in an undifferentiated state through inhibition of the proadipogenic transcription factors CCAAT- enhancer binding protein $\alpha$ and peroxisome proliferator-activated receptor $\gamma$ [34]. Therefore, the higher expression of Pparg in undifferentiated $\mathrm{ASCs}^{\mathrm{ZDF}}$ may be a result of the reduced Wnt (also known as $W n t 2$ ) expression, again indicating a greater predisposition to adipogenic differentiation in $\mathrm{ASC}^{\mathrm{ZDF}}$.

Beside the Notch and Wnt pathways, the fate of MSCs is also determined by fibroblast growth factor (FGF) signalling [35]. Gene expression analysis also revealed an effect of diabetes on the FGF pathway, which has been implicated in cell survival, proliferation, pluripotency and the lineage determination of stem cells [36, 37]. FGF2 supports the osteogenic and chondrogenic differentiation potential of MSCs and a marked increase in adipocytes has been observed in BMSC cultures from $\mathrm{Fg}_{2} 2^{-/}$animals [37]. The effect on FGF signalling might have contributed to the differences in adipogenic and osteogenic differentiation observed between $\mathrm{ASCs}^{\mathrm{ZDF}}$ and $\mathrm{ASCs}{ }^{\mathrm{ZLC}}$.

It has been reported that advanced glycation end-products interfere with in vitro differentiation of MSCs into adipogenic, chondrogenic and osteogenic lineages [38]. Pre-adipocytes of type 2 diabetes participants have shown decreased adipogenic differentiation capacity [39] and a high glucose concentration is a potent inhibitor of BMPC differentiation into osteoblastic
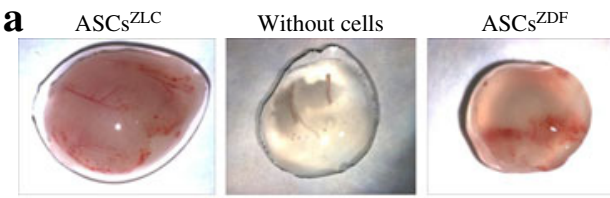

Without cells
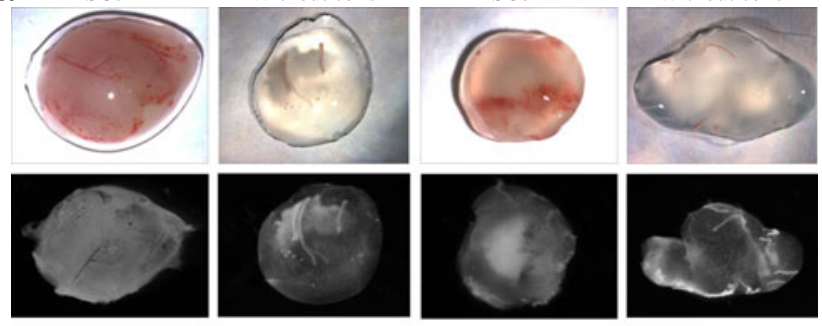

b

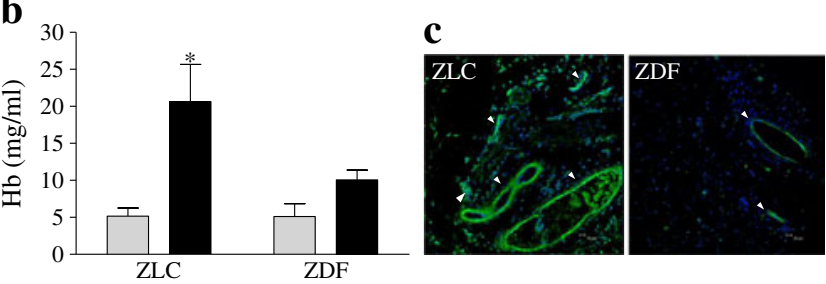

Fig. 6 Angiogenesis assay in vivo. (a) Macroscopic view of representative blood vessels formed in Matrigel plugs in vivo ( $n=4$ per group). Plugs were visualised and photographed with a Zoom 70XL (Optem; Fairport, NY, USA) coupled to a camera (Retiga 1300i Fast Camera; Surrey, BC, Canada), or with a fluorescence stereomicroscope (M165 FC; Leica; Wetzlar, Germany) (top to bottom row, respectively). (b) Quantification of haemoglobin $(\mathrm{Hb})$ concentration using Drabkin's method. Grey bars, Matrigel without cells; black bars, Matrigel with ASCs. ${ }^{*} p<0.05$ for Matrigel with ASCs vs Matrigel without cells. (c) Immunofluorescence staining for von Willebrand factor (green) and nucleus (blue). White arrowheads, neovessel formation 

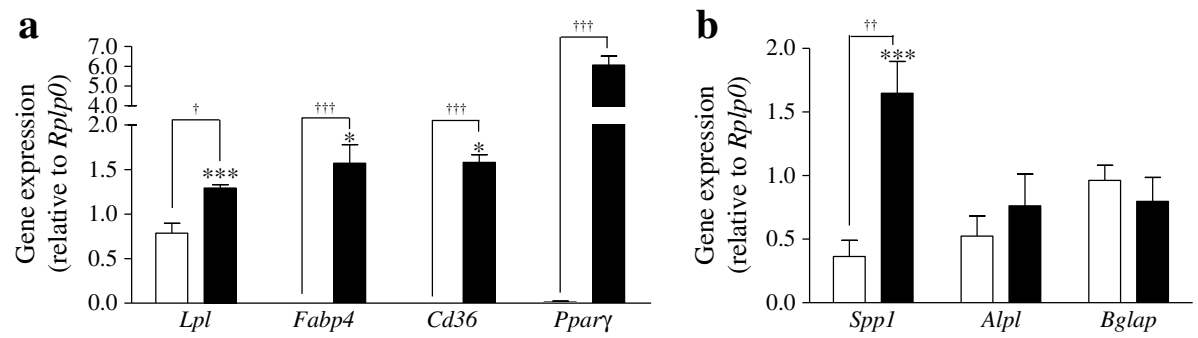

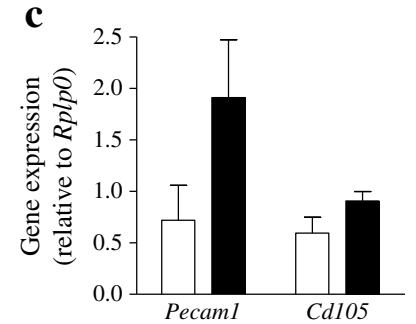

Fig. 7 Differentiation potential of ASCs from ChDMII rats. To evaluate the differentiation potential of ASCs from ChDMII rats, cells were induced to differentiate into adipocytes $(\mathbf{a}, \mathbf{d})$, osteoblasts $(\mathbf{b}, \mathbf{e})$ and ECs $(\mathbf{c}, \mathbf{f})$. White bars, undifferentiated ASCs ${ }^{\text {ChDMII}}$; black bars, differentiated ASCs ${ }^{\text {ChDMII }}$. The acquisition of a lineage-specific phenotype was evaluated by real-time PCR. Each data point is presented as gene

lineages [40]. In agreement with these studies, we observed impaired osteogenic differentiation of ASCs ${ }^{\mathrm{ZDF}}$. However, we found that the adipogenic differentiation potential of ASCs ${ }^{\mathrm{ZDF}}$ was higher than that of $\mathrm{ASCs}^{\mathrm{ZLC}}$. Aguiari et al show that glucose-enriched medium induces the adipogenic differentiation of stem cells [41]. We propose that the effect of diabetes on Notch, Wnt and FGF signalling is the cause of ASCs ${ }^{\text {ZDF }}$ commitment to the adipogenic lineage compared with $\mathrm{ASCs}^{\mathrm{ZLC}}$. Therefore, the effects of type 2 diabetes in major signalling pathways implicated in the maintenance of the undifferentiated state might compromise the plasticity and self-renewal of ASCs and, consequently, the strategies for tissue regeneration in diabetic patients. Further studies will be required to confirm the involvement of these signalling pathways in the commitment of $\mathrm{ASCs}^{\mathrm{ZDF}}$ to an adipogenic lineage.

To test whether diabetes affects the EC differentiation potential of ASCs, we induced their differentiation towards ECs. Although both differentiated ASC groups were able to develop capillary-like networks, the ability of ASCs ${ }^{\mathrm{ZDF}}$ was significantly impaired. We also performed in vivo plug angiogenesis assays to study the ability of ASCs to form new vessels. Consistent with their in vitro EC differentiation capacity, $\mathrm{ASCs}^{\text {ZDF }}$ had an impaired ability to stimulate the formation of an extended network of microvessels in the implanted plug. Several studies have shown a role of the Notch and Wnt pathways in angiogenesis [42-44]. Notch signalling is required for angiogenesis in ischaemia models, and Dll1, the expression of which was lower in ASCs ${ }^{\mathrm{ZDF}}$, is essential for postnatal angiogenesis [45], while jagged-1 expression levels relative to the reference gene. The results $(\mathbf{a}-\mathbf{c})$ are means $\pm \mathrm{SEM} ; n=4$ per group, in technical duplicates. No significant differences were observed between ASCs ${ }^{\mathrm{ChDMII}}$ and $\mathrm{ASCs}^{\mathrm{ZDF}}{ }^{*} p<0.05$ and $* * * p<0.001$ for differentiated ASCs ${ }^{\text {ChDMII }}$ vs differentiated $\mathrm{ASCs}^{\mathrm{ZLC}} ;{ }^{\dagger} p<0.05,{ }^{\dagger \dagger} p<0.01$ and ${ }^{\dagger \dagger} p<0.001$ for differentiated $\mathrm{ASCs}^{\text {ChDMII }}$ vs undifferentiated ASCs ${ }^{\text {ChDMII }}$

(Jagl), which we also found downregulated, enhances angiogenesis [46]. Recent reports have underlined the important role of the Wnt system in vascular morphogenesis and in organ-specific EC differentiation [47]. It has also been reported that anti-angiogenic factors, such as collagen, type XVIII, alpha 1, inhibit Wnt signalling activity [48]. Our results for gene expression analysis revealed a significant increase in expression of Coll8al in ASCs ${ }^{\mathrm{ZDF}}$. Therefore, the inhibition of signalling, with the increase in anti-angiogenic factors, might be responsible for the impaired capacity of ASCs ${ }^{\text {ZDF }}$ to develop newly formed microvessels.

Finally, because leptin is involved in regulating MSC differentiation $[49,50]$, we investigated another model of diabetes in addition to the ZDF leptin receptor-deficient model. Its gene expression profile, as well as differentiation potential, showed a similar pattern to $\mathrm{ASCs}^{\mathrm{ZDF}}$ and $\mathrm{ASCs}^{\mathrm{ChDMII}}$. Therefore, we can conclude that the impaired differentiation potential observed in $\mathrm{ASCs}^{\mathrm{ZDF}}$ is independent of leptin receptor deficiency.

In summary, this is to our knowledge the first report to analyse the effect of diabetes on molecules involved in the maintenance of pluripotency and self-renewal of ASCs. Our results indicate that the effects of diabetes on main signalling pathways implicated in the maintenance of an undifferentiated state are the cause of the greater ASCs ${ }^{\mathrm{ZDF}}$ commitment to an adipogenic lineage, compared with $\mathrm{ASCs}^{\mathrm{ZLC}}$, and that this leads to a gradual depletion of the ASC pool.

For the first time, we demonstrate that the EC differentiation potential and the ability of ASCs ${ }^{\mathrm{ZDF}}$ to develop capillarylike networks in vitro and in vivo are significantly impaired 
compared with $\mathrm{ASCs}^{\mathrm{ZLC}}$. Functional results, as well as gene expression and subsequent in silico analysis show that the benefits of using ASCs derived from diabetic patients might be limited in angiogenic therapies. Collectively, our data also help to explain the reduced spontaneous self-repair of hypoxic and ischaemic damage in diabetic patients.

Acknowledgements The authors would like to thank J. Crespo (Flow Cytometry Department, Cardiovascular Research Center, CSIC-ICCC, Barcelona, Spain) for his technical help with flow cytometric analysis and M. Sanchez (Cardiovascular Research Center, CSIC-ICCC, Barcelona, Spain) for her excellent technical assistance.

Funding This work was supported by research grants from the Spanish Ministry of Health-Instituto de Salud Carlos III (CIBERobn-CB06/03 and RETIC TERCEL RD/06/0010/0017), the Spanish Ministry of Science and Innovation (PNS SAF2010/16549) and the Government of Catalonia (CTP-ITT 2009). RFL is recipient of a Sara Borrell contract from the Spanish Ministry of Science and Innovation-Instituto de Salud Carlos III. MTB has received a fellowship from Institut Català de Ciències Cardiovasculars. MT was a Postdoctoral Fellow of Fundacion de Investigacion Cardiovascular and a Juan de la Cierva investigator (Spanish Ministry of Science and Innovation, MICINN). GV is a Ramon y Cajal investigator (Spanish Ministry of Science and Innovation, MICINN).

Duality of interest The authors declare that there is no duality of interest associated with this manuscript.

Contribution statement RFL, GV and LB contributed to the study conception and design. RFL, MTB and MT contributed to the acquisition of data. RFL and MTB contributed to the analysis of data. RFL, MTB and LB contributed to the interpretation of data. RFL was responsible for writing the manuscript. All authors contributed to the revision of the manuscript and approved the final version to be published.

\section{References}

1. Pittenger MF, Martin BJ (2004) Mesenchymal stem cells and their potential as cardiac therapeutics. Circ Res 95:9-20

2. Van der Bogt KEA, Sheikh AY, Schrepfer S et al (2008) Comparison of different adult stem cell types for treatment of myocardial ischemia. Circulation 118:S121-S129

3. Kamihata $H$, Matsubara $H$, Nishiue $T$ et al (2001) Implantation of bone marrow mononuclear cells into ischemic myocardium enhances collateral perfusion and regional function via side supply of angioblasts, angiogenic ligands, and cytokines. Circulation 104: 1046-1052

4. Orlic D, Kajstura J, Chimenti S et al (2001) Bone marrow cells regenerate infarcted myocardium. Nature 410:701-705

5. Cleland JGF, Fremantle N, Coletta AP, Clark AL (2006) Clinical trials update from the American Heart Association: REPAIR-AMI, ASTAMI, JELIS, MEGA, REVIVE-II, SURVIVE, and PROACTIVE. Eur J Heart Fail 8:105-110

6. Gimble JM, Guilak F, Bunnell BA (2010) Clinical and preclinical translation of cell-based therapies using adipose tissue-derived cells. Stem Cell Res Ther 1:19

7. Tepper OM, Galiano RD, Capla JM et al (2002) Human endothelial progenitor cells from type II diabetics exhibit impaired proliferation, adhesion, and incorporation into vascular structures. Circulation 106: 2781-2786
8. Li TS, Furutani A, Takahashi M et al (2006) Impaired potency of bone marrow mononuclear cells for inducing therapeutic angiogenesis in obese diabetic rats. Am J Physiol Heart Circ Physiol 290: H1362-H1369

9. Fadini GP, Sartore S, Schiavon M et al (2006) Diabetes impairs progenitor cell mobilization after hindlimb ischaemia-reperfusion injury in rats. Diabetologia 49:3075-3084

10. Sharpless NE, DePinho RA (2007) How stem cells age and why this makes us grow old. Nat Rev Mol Cell Biol 8:703-713

11. Friedenstein AJ, Piatetzky-Shapiro II, Petrakova KV (1966) Osteogenesis in transplants of bone marrow cells. J Embryol Exp Morpholog 16: 381-390

12. Dominici M, Le Blanc K, Mueller E et al (2006) Minimal criteria for defining multipotent mesenchymal stromal cells. The International Society for Cellular Therapy position statement. Cytotherapy 8:315-317

13. Chamberlain G, Fox J, Ashton B, Middleton J (2007) Concise review: mesenchymal stem cells: their phenotype, differentiation capacity, immunological features, and potential for homing. Stem Cells 25:2739-2749

14. Da Silva ML, Chagastelles PC, Nardi NB (2006) Mesenchymal stem cells reside in virtually all post-natal organs and tissues. J Cell Sci 119:2204-2213

15. Zuk PA, Zhu M, Ashjian P et al (2002) Human adipose tissue is a source of multipotent stem cells. Mol Biol Cell 13:4279-4295

16. Gimble JM, Katz AJ, Bunnell BA (2007) Adipose-derived stem cells for regenerative medicine. Circ Res 100:1249-1260

17. Zhu Y, Liu T, Song K, Fan X, Ma X, Cui Z (2008) Adipose-derived stem cell: a better stem cell than BMSC. Cell Biochem Funct 26: 664-675

18. Schäffler A, Büchler C (2007) Concise review: adipose tissuederived stromal cells - basic and clinical implications for novel cell-based therapies. Stem Cells 25:818-827

19. Madonna R, Geng YJ, de Caterina R (2009) Adipose tissue derived stem cells: characterization and potential for cardiovascular repair. Arterioscler Thromb Vasc Biol 29:1723-1729

20. Mizuno H, Tobita M, Uysal AC (2012) Adipose-derived stem cells as a novel tool for future regenerative medicine. Stem Cells 30:804-810

21. Clark JB, Palmer CJ, Shaw WN (1983) The diabetic Zucker fatty rat. Proc Soc Exp Biol Med 173:68-75

22. Masiello P, Broca C, Gross R et al (1998) Experimental NIDDM: development of a new model in adult rats administered streptozotocin and nicotinamide. Diabetes 47:224-229

23. Golzelniak K, Janke J, Engeli S, Sharma AM (2001) Validation of endogenous controls for gene expression studies in human adipocytes and preadipocytes. Horm Metab Res 33:625-627

24. Li YM, Schilling T, Benisch P et al (2007) Effects of high glucose on mesenchymal stem cell proliferation and differentiation. Biochem Biophys Res Commun 363:209-215

25. Nichols J, Zevnik B, Anastassiadis K et al (1998) Formation of pluripotent stem cells in the mammalian embryo depends on the POU transcription factor Oct4. Cell 95:379-391

26. Boiani M, Schöler HR (2005) Regulatory networks in embryoderived pluripotent stem cells. Nat Rev Mol Cell Biol 6:872-884

27. Riekstina U, Cakstina I, Parfejevs V et al (2009) Embryonic stem cell markers expression pattern in human mesenchymal stem cells derived from bone marrow, adipose tissue, heart and dermis. Stem Cell Rev Rep 5:378-386

28. Park IH, Zhao R, West JA et al (2008) Reprogramming of human somatic cells to pluripotency with defined factors. Nature 451:141-146

29. Radtke F, Raj K (2003) The role of Notch in tumorigenesis: oncogene or tumor suppressor? Nat Rev Cancer 3:765-767

30. Nichols AM, Pan Y, Herreman A et al (2004) Notch pathway is dispensable for adypocite specification. Genesis 40:40-44 
31. Garcés C, Ruiz-Hidalgo MJ, Font de Mora J et al (1997) Notch-1 controls the expression of fatty acid-activated transcription factors and is required for adipogenesis. J Biol Chem 272:29729-29734

32. Ugarte F, Ryser M, Thieme $S$ et al (2009) Notch signalling enhances osteogenic differentiation while inhibiting adipogenesis in primary human bone marrow stromal cells. Exp Hematol 37:867-875

33. Huang Y, Yang X, Wu Y et al (2010) $\gamma$-Secretase inhibitor induces adipogenesis of adipose-derived stem cells by notch regulation of Notch and PPAR $\gamma$. Cell Prolif 43:147-156

34. Laudes M (2011) Role of Wnt signalling in the determination of human mesenchymal stem cells into preadipocytes. J Mol Endocrinol 46:65-72

35. Lanner F, Rossant J (2010) The role of FGF/Erk signalling in pluripotent cells. Development 137:3351-3360

36. Zaragosi LE, Ailhaud G, Dani C (2006) Autocrine fibroblast growth factor 2 signalling is critical for self-renewal of human multipotent adipose-derived stem cells. Stem Cells 24:2412-2419

37. Xiao L, Sobue T, Esliger A et al (2010) Disruption of the Fgf2 gene activates the adipogenic and suppresses the osteogenic program in mesenchymal marrow stromal stem cells. Bone 47:360-370

38. Kume S, Kato S, Yamagishi S et al (2005) Advanced glycation endproducts attenuate human mesenchymal stem cells and prevent cognate differentiation into adipose tissue, cartilage, and bone. J Bone Miner Res 20:1647-1658

39. Van Tienen FHJ, van der Kallen CJH, Lindsey PJ, Wanders RJ, van Grevenbroek MM, Smeets HJM (2011) Preadypocytes of type 2 diabetes subjects display an intrinsic gene expression profile of decreased differentiation capacity. Int J Obes 35:1154-1164
40. Gopalakrishnan V, Vignesh RC, Arunakaran J, Aruldhas MM, Srinivasan N (2006) Effects of glucose and its modulation by insulin and estradiol on BMSC differentiation into osteoblastic lineages. Biochem Cell Biol 84:93-101

41. Aguiari P, Leo S, Zavan B et al (2008) High glucose induces adipogenic differentiation of muscle-derived stem cells. PNAS 105: 1226-1231

42. Bicknell R, Harris AL (2004) Novel angiogenic signalling pathways and vascular targets. Annu Rev Pharmacol Toxicol 44:219-238

43. Zerlin M, Julius MA, Kitajewski J (2008) Wnt/Frizzled signalling in angiogenesis. Angiogenesis 1:63-69

44. Phng LK, Gerhardt H (2009) Angiogenesis: a team effort coordinated by Notch. Dev Cell 16:196-208

45. Limbourg A, Ploom M, Elligsen D et al (2007) Notch ligand deltalike 1 is essential for postnatal arteriogenesis. Circ Res 100:363-371

46. Benedito R, Roca C, Sörensen I et al (2009) The Notch ligands D114 and Jagged1 have opposing effects on angiogenesis. Cell 137:1124 1135

47. Dejana E (2010) The role of Wnt signalling in physiological and pathological angiogenesis. Circ Res 107:943-952

48. Zhang B, Ma J (2010) Wnt pathway antagonists and angiogenesis. Protein Cell 1:898-906

49. Chang YJ, Shih DT, Tseng CP, Hsieh TB, Lee DC, Hwang SM (2006) Disparate mesenchyme-lineage tendencies in mesenchymal stem cells from human bone marrow and umbilical cord blood. Stem Cells 24:679-685

50. Scheller EL, Song J, Dishowitz MI, Soki FN, Hankenson KD, Krebsbach PH (2010) Leptin functions peripherally to regulate differentiation of mesenchymal progenitor cells. Stem Cells 28:1071-1080 